\title{
Review Article \\ The Role of Epigenetic Alterations in Papillary Thyroid Carcinogenesis
}

\author{
Ogechukwu P. Eze, ${ }^{1,2}$ Lee F. Starker, ${ }^{1,2}$ and Tobias Carling, ${ }^{1,3}$ \\ ${ }^{1}$ Department of Surgery, Yale University School of Medicine, 333 Cedar Street, TMP202, Box 208062, New Haven, \\ CT 06520-8062, USA \\ ${ }^{2}$ Yale Endocrine Neoplasia Laboratory, Yale University School of Medicine, 333 Cedar Street, TMP202, Box 208062, New Haven, \\ CT 06520-3206, USA \\ ${ }^{3}$ The Cancer Genetics Program, Yale Cancer Center, Yale University School of Medicine, New Haven, CT 06520-8028, USA
}

Correspondence should be addressed to Tobias Carling, tobias.carling@yale.edu

Received 14 January 2011; Accepted 4 April 2011

Academic Editor: Ana O. Hoff

Copyright (C) 2011 Ogechukwu P. Eze et al. This is an open access article distributed under the Creative Commons Attribution License, which permits unrestricted use, distribution, and reproduction in any medium, provided the original work is properly cited.

\begin{abstract}
Papillary thyroid carcinoma (PTC) accounts for over $80 \%$ of all thyroid malignancies. The molecular pathogenesis remains incompletely clarified although activation of the RET fusion oncogenes, and RAS and BRAF oncogenes, has been well characterized. Novel technologies using genome-wide approaches to study tumor genomes and epigenomes have provided great insights into tumor development. Growing evidence shows that acquired epigenetic abnormalities participate with genetic alterations to cause altered patterns of gene expression/function. It has been established beyond doubt that promoter cytosine methylation in CpG islands, and the subsequent gene silencing, is intimately involved in cancer development. These epigenetic events very likely contribute to significant variation in gene expression profiling, phenotypic features, and biologic characteristics seen in PTC. Hypermethylation of promoter regions has also been analyzed in PTC, and most studies have focused on individual genes or a small cohort of genes implicated in tumorigenesis.
\end{abstract}

\section{Background}

Thyroid carcinoma is the most common endocrine malignancy. Papillary thyroid cancer (PTC) accounts for over $90 \%$ of thyroid malignancies [1]. With improved diagnostic techniques, papillary thyroid carcinoma is identified much more frequently than in the past [2]. Currently, ways to preoperatively identify patients with invasive thyroid cancer include asymptomatic disease with concomitant vocal cord dysfunction or subglottic/tracheal mass, recurrent disease in the central compartment, symptomatic disease with voice changes, dyspnea, hemoptysis, or dysphagia, and finally documented invasive disease based on preoperative imaging [3]. More papillary thyroid cancers are diagnosed as microcarcinomas; therefore, molecular methods of detecting aggressive disease will aid in treatment planning.

The majority of genetic alterations in thyroid cancer exert their oncogenic actions at least partially through the activation of the MAP kinase/ERK pathway. Constitutive activation of the MAP kinase/ERK pathway leads to tumorigenesis by upregulating cell division and proliferation [4]. Activation of this pathway is a common and important mechanism in the genesis and progression of human cancers. When constitutively activated, the MAP kinase pathway leads to tumorigenesis [4]. Alterations of RET/PTC, BRAF, and RAS genes are linked to papillary thyroid tumorigenesis.

RET proto-oncogene encodes a cell membrane receptor tyrosine kinase. Ligands of the kinase are of the glialcell line-derived neurotrophic factor (GDNF) family which cause the receptors to dimerize upon binding, leading to autophosphorylation of tyrosine residues and initiation of the MAP kinase/ERK pathway signaling cascade [5]. High expression of RET in parafollicular C-cells of the thyroid gland is consistent with its role in the development of neural crest-derived cell lineages. This high expression of RET does not normally occur in thyroid follicular cells; 
however, RET activation in these cells occurs by fusion of the 3' tyrosine domain of RET to the 5' portion of constitutively expressed genes. The most common RET/PTC rearrangements seen in PTC are RET/PTC1 (fusion with $\mathrm{H} 4$ gene) and RET/PTC3 (fusion with NCOA4 gene). Prevalence of RET/PTC rearrangements in PTC is greatest in populations exposed to radiation (60-70\%). In the general population, the prevalence is higher in children [6]. Activation of RET/PTC results in downregulation of thyroglobulin and sodium iodide importer genes, which are thyroid specific, and thyroid follicular cell differentiation. Wild-type and truncated forms of RET/PTC activate the PI3K/AKT pathway leading to tumorigenesis $[6,7]$.

Mutations of BRAF, a serine-threonine kinase and downstream signaling molecule of RAS and RET, are potent activators of the MAP kinase/ERK pathway $[4,8]$. These missense mutations of the BRAF gene, located on chromosome 7, occur in the kinase domain with the T1799A transversion mutation that results in a single amino acid substitution of valine to glutamic acid (V600E) accounting for $80-90 \%$ of BRAF activating mutations $[6,9]$. The V600E mutation is thought to mimic phosphorylation in the activation segment of BRAF by inserting a negatively charged residue adjacent to an activating phosphorylation site [4].

BRAF V600E occurs as a sporadic mutation in thyroid cancer $[9,10]$ and is restricted to papillary and anaplastic or poorly differentiated carcinomas [11]. Prevalence of the mutation is reported in papillary thyroid cancer at 35$40 \%$, with a significantly higher prevalence in males than females [9]. The rate of BRAF mutation in PTC is the second highest to that in melanomas $(60 \%)$ and is much higher than other cancers such as colorectal adenocarcinomas (5-10\%) and lung cancers (1.8\%) [9]. BRAF V600E correlates with poorer clinicopathologic outcomes defined as extrathyroidal extension, lymph node metastasis, and advanced tumor grade (III/IV) at presentation and is prognostic of tumor recurrence [12-15].

Activating mutations of the three RAS oncogenes (HRAS, K-RAS, and N-RAS) occur in thyroid tumors; however, their prevalence depending on histology of the tumors has been controversial [16]. Early studies demonstrated that RAS mutations were more frequent in follicular tumors than papillary thyroid cancers, in addition to different patterns of mutations occurring in the two types. Indeed, mutations in up to $50 \%$ on microfollicular adenoma further supported the idea that RAS oncogene activation was an early event in follicular thyroid tumorigenesis. More recent studies have reported varying incidences of RAS mutations in thyroid tumors $(0-50 \%$ in PTC, $0-85 \%$ in adenomas, $14-$ $62 \%$ in FTC, and $0-60 \%$ in anaplastic carcinomas). Some investigators find no correlation between RAS mutation isoforms and tumor pathology, while others report a higher frequency of mutations in codon61 of H-RAS and N-RAS in FTC and poorly differentiated carcinomas.

Although not confirmed by similar studies [10], 38\% of BRAF-positive papillary thyroid tumors showed RET/PTC rearrangement [9], contrary to reports that BRAF V600E mutation does not occur with RET/PTC or RAS mutations in cancer [10]. Concurrent RET/PTC and BRAF mutations has also recently been reported in papillary thyroid cancer [17]. The low oncogenic potential of both BRAF and RET/PTC1 suggest that both mutations occurring in the same pathway are not necessarily redundant but may cooperate in papillary thyroid tumorigenesis. Indeed, RET/PTC1 and RAS mutations have been shown to synergistically lead to tumorigenesis [9].

The molecular pathogenesis of PTC thus remains incompletely clarified. With respect to gene alterations, papillary thyroid cancers have relatively low rates of loss of heterozygosity, with no specific region displaying a particularly high prevalence when compared to follicular thyroid cancers [18]. Like RET, BRAF, or RAS mutations, other molecular alterations are thought to be essential for the induction of papillary thyroid cancer. Epigenetic events very likely contribute to significant variation in gene expression profiling, phenotypical features, and biologic characteristics seen among papillary thyroid carcinoma [19].

\section{Epigenetics Mechanisms Involved in Tumorigenesis}

Epigenetic silencing of regulatory genes is part of the global genomic alterations in cancer that alter pathways relevant to stem-cell growth and differentiation. Epigenetic silencing mechanisms include covalent modifications of chromatin, DNA cytosine methylation, noncoding RNAs, and nucleosome remodeling [20]. It has been proposed that epigenetic abnormalities may play a seminal role in the earliest steps in tumorigenesis [21-24]. Epigenetic changes may act in concert with genetic changes resulting in tumorigenesis, because they are mitotically heritable. The high degree of mitotic stability of silencing plus the progressive nature by which it is achieved makes pathological silencing of growth controlling and other genes essential to carcinogenesis.

Patterns of DNA methylation are linked to gene expression; for example, methylation in a gene promoter region generally correlates with a silenced gene [25]. DNA methylation, the DNA methyltransferase (DNMT) catalyzed addition of methyl group to cytosine ring, is restricted to cytosines that precede a guanosine in the DNA sequence (the CpG dinucleotide) in humans and other mammals [25]. The distribution of $\mathrm{CpG}$ dinucleotides in the genome is unusually asymmetric, occurring in small clusters called "CpG islands". The CpG islands are often in promoter regions of genes and are usually unmethylated regardless of the transcriptional state. This highlights the importance of DNA methylation for gene expression, especially in transcriptional silencing [25].

Aberrant DNA methylation plays a strong role in tumorigenesis. Global hypomethylation of intergenic $\mathrm{CpG}$ dinucleotides and regional hypermethylation of $\mathrm{CpG}$ islands in promoter regions are characteristic hallmarks of many cancers [24]. The impact of hypermethylation on tumorigenesis is further illustrated by the silencing of multiple tumor suppressor genes, thereby contributing to the hallmarks of carcinogenesis which include evading apoptosis (P53, p14ARF, BNIP3 and Caspase-8), insensitivity to antigrowth signals (p16INK4a and miR-124a), sustained angiogenesis (TIMP3 and TSP1), limitless replicative potential 
(hTERT), and tissue invasion and metastasis (E-cadherin and LIMS2).

Transcriptional silencing is also a result of chromatin compaction due to convergence of DNA methylation and histone modifications. Methylated DNA recruits methylbinding proteins (MBDPs), which have methyl-CpG-binding domains (MBD), to hypermethylated DNA. MBDPs associate with histone deacetylases, resulting in chromatin remodeling and gene silencing. In addition to these mechanisms of silencing, histone methyltransferase (HMTs) repress transcription by methylation of lysine 9 of histone 3 (H3K9) or lysine 27 of histone 3 (H3K27). An excellent review of epigenetic modifications of chromatin is provided by Iacobuzio-Donahue [26].

The influence of epigenetic events on tumorigenesis is well illustrated by the evolution of colon cancer, in which risk factors for common cancers such as aging and inflammation are shown to cause expansions in either normal colon epithelial stem cells or precursor cells derived from them. Epigenetic gatekeepers such as cyclin-dependent kinase inhibitor $2 \mathrm{~A}(\mathrm{CDKN} 2 \mathrm{~A} / \mathrm{p} 16)$, secreted frizzled-related protein (SFRP), GATA-binding protein 4 and 5 (GATA-4 and -5), and adenomatous polyposis of the colon (APC) prevent early tumor progression in colon cancer. Normal epigenetic modulation of these gatekeeper genes allows them to prevent stem/precursor cells from becoming immortalized during periods of chronic stresses and renewal pressures on cell systems. APC is a classically mutated tumor suppressor gene in colon cancer, which is also inactivated by epigenetic mechanisms [27]. Epigenetic silencing of one allele serves as a second-hit in Knudson's hypothesis for tumor suppressor gene inactivation when paired which mutations on the other allele [21].

Like APC, loss of p16 can be epigenetically mediated, permitting expanding cells to develop genomic instability $[28,29]$ and further epigenetic gene-silencing events [30]. Its loss is seen in subsets of preinvasive stages of colon and other cancers [30]. Finally, GATA-4 and -5 transcription factor genes, important for both embryonic gastrointestinal epithelial development and for maturation in adults, are epigenetically silenced in about half of all the preinvasive and invasive lesions for colon cancer [31]. This can hamper differentiation and promote precursor cell expansion.

The wingless-type MMTV integration site (Wnt) pathway activation also illustrates how multiple epigenetic events may act in concert to affect a single-cell pathway. Inappropriate silencing of these genes leads to abnormal activation of the Wnt pathway, which plays a canonical role in colon tumorigenesis [23]. These genes are independently affected by epigenetic events but result in Wnt activation. Four genes in the SFRP family encoding proteins that antagonize the action of the Wnt ligand at the cell membrane are hypermethylated simultaneously in the majority of preinvasive lesions for colon cancer. Upregulation of the survival protein Sirtuin 1 (SIRT1) also results in Wnt pathway activation. SIRT1 is upregulated as a result of loss of the transcription factor hypermethylated in cancer 1 (HIC1) via hypermethylation in early preinvasive lesions in colon cancer as well as other types of cancer [23]. Loss of HIC1 also results in additional gene silencing events as well as downregulation of tumor protein 53 (p53).

Loss of DNA methylation results in the weakening of transcriptional repression in normally silent regions of the genome resulting in harmful expression of inserted or normally silenced genes, and loss of functional stability of chromosomes. It has been established that covalent histone modification is linked to DNA methylation. Cytosine methylation attracts methylated DNA-binding proteins and histone deacetylases to methylated $\mathrm{CpG}$ islands during chromatin compaction and gene silencing [32, 33]. In addition to epigenetic modification of transcriptional start sites, there is evidence for more global changes in chromatin structure. For instance, there is an overall decrease in the 5-methylcytosine content of cancer genomes that is reflected as hypermethylation in CpG islands [34]. The consistently observed hypermethylation is due to a change in 5-methylcytosine distributions rather than an overall increase in total amount of methylation. It has also been observed that large stretches of DNA can become abnormally methylated in cancer.

\section{Epigenetics of Papillary Thyroid Carcinogenesis}

Quantitative analysis of promoter hypermethylation in thyroid cancer has involved RASSF1A, TSHR, RAR $\beta 2$, DAPK, S100, p16, CDH1, CALCA, TIMP3, TGF- $\beta$, and GSTpi [35]. Hypermethylation of 2 or more markers (RASSF1A, TSHR, RAR $\beta 2$, DAPK, CDH1, TIMP3, and TGF- $\beta$ ) was detectable in $25 \%$ of thyroid hyperplasias, $38 \%$ of adenomas, $48 \%$ of thyroid cancers, and $100 \%$ of cell lines. Rank correlation analysis of marker hypermethylation suggests that a subset of the markers were hypermethylated in concert, which may represent a thyroid-specific regulatory process [35]. Additionally, a positive correlation was observed between BRAF mutation and $R A R \beta 2$ and a negative correlation between BRAF mutation and RASSF1A [35].

Investigation of DNA methylation in PTC has been predominantly restricted to individual candidate tumor suppressor genes and genes known for their role in thyroid function, using locus specific nonquantitative methods. BRAF, RASSFIA, TSHR, ECAD, NIS-L, ATM, DAPK, SLC5A8, TIMP3, and RAR $\beta 2$ have been analyzed for DNA methylation. Promoter hypermethylation of TSHR, NIS-L, $\mathrm{ATM}$, and ECAD has been demonstrated in 34-59\% [36, 37], $22 \%, 50 \%$, and $56 \%$ of patients with papillary thyroid cancer, respectively [37].

3.1. Thyroid-Stimulating Hormone Receptor (TSHR) and Sodium Iodide Symporter (NIS). TSHR stimulates several key steps in thyrocyte concentration of iodine, including uptake by NIS and oxidation before incorporation into thyroglobulin by thyroid peroxidase [36]. The methylation status of the NIS and TSHR promoter regions are important, because these genes are specific to the thyroid and play a role in the uptake of iodine and normal cellular function [37]. Promoter hypermethylation resulting in decreased expression of TSHR and NIS may result in a decreased ability to concentrate iodine, rendering ablative doses 
of ${ }^{131}$ I ineffective [37]. Promoter hypermethylation of TSHR is reported in $34-59 \%[36,37]$ of patients with papillary thyroid cancer. NIS mRNA expression has been shown to be decreased in thyroid cancers $[37,38]$, and this has been proposed to be secondary to methylation of the promoter region [32, 37]. The NIS-L region within the promoter was shown to be hypermethylated in $22 \%$ (7/32) of patients with papillary thyroid cancer [37] but was not methylated in surrounding histologically benign tissue.

3.2. E-Cadherin (ECAD). E-cadherin complexes with catenins to promote $\mathrm{Ca}^{2+}$-dependent, homotypic cell-to-cell adhesion and to establish normal epithelial tissue architecture [39]. Disruption of the E-cadherin/catenin complex contributes to tumor metastasis, and decreased expression of E-cadherin is observed in advanced stage, poorly differentiated carcinomas [39]. Promoter hypermethylation has been demonstrated in multiple human cancers, including papillary thyroid cancer in $56 \%(18 / 32)$ of patients [37].

3.3. Ataxia Telangiectasia Mutated (ATM). ATM is a member of the phosphatidylinositol 3-kinase family of proteins that respond to DNA damage by phosphorylating key substrates (p53 and BRCA1) involved in DNA repair and/or cell cycle control [40-42]. Hypermethylation of ATM promoter was observed in 50\% (16/32) of patients with papillary thyroid cancer analyzed [37].

3.4. Apical Iodide Transporter (AIT). The thyroid apical iodide transporter AIT encoded by the SCL5A8 gene has been defined as a sodium-coupled transporter of shortchain fatty acid. It is thought that AIT may be involved in the passive transport of iodide from thyrocyte to the follicle lumen $[43,44]$. Expression of SCL5A8 is decreased in thyroid cancers compared to other iodide transporters [43] and is expressed abundantly in colon cancer, functioning as a tumor-suppressor gene. Silencing of SLC5A8 occurs by promoter hypermethylation in about $50 \%$ of colon cancer cell lines and primary colon cancers. Decreased expression of SLC5A8 observed in classical variant of papillary thyroid cancer is linked to hypermethylation of exon 1 of the gene [44].

Hypermethylation occurred in 33\% (76/231) of PTC and was associated with extrathyroidal invasion (40\%) and multifocality (40\%) [45]. This epigenetic event is thought occur at a later stage in papillary thyroid cancer and specific of the classical variant; therefore, it may be secondary to other genetic alterations occurring selectively in the tumor type [44]. Indeed, SLC5A8 and BRAF discriminate the classical variant PTC, supporting the argument. In addition, a strong association between low SLC5A8 expression and the presence of BRAF V600E [44] or advanced clinicopathologic features [45] suggests a link in the progression to more aggressive papillary thyroid cancer.

3.5. The Tissue Inhibitor of Metalloproteinase 3 (TIMP3). TIMP3 is one of 4 tissue inhibitors of metalloproteinase thought to inhibit growth, angiogenesis, invasion, and metastasis in several human cancers $[45,46]$. TIMP3 inhibits vascular endothelial factor-(VEGF-) mediated angiogenesis by blocking the binding of VEGF to VEGF receptor-2, thereby inhibiting downstream signaling and angiogenesis [46]. Promoter hypermethylation, and downregulation of TIMP3 expression, is observed in various human cancers [45, 47-49]. Hypermethylation in PTC occurred in 53\% of tumors analyzed and was associated with extrathyroidal invasion (38\%), lymph node metastasis (43\%), and multifocality (49\%).

3.6. Death-Associated Protein Kinase (DAPK). DAPK is a calcium/calmodulin-dependent serine threonine kinase protein with a proapoptotic, tumor-suppressor function $[45,50]$. The DAPK gene is silenced by hypermethylation in several human cancers $[45,51,52]$, including thyroid cancer, and its expression has been shown to be a useful marker for cancer prognosis [50]. In addition to aberrant DNA methylation, chromatin immunoprecipitation analysis demonstrated that histone deacetylation of the 5' $\mathrm{CpG}$ island is involved in gastrointestinal malignancies [51]. In papillary thyroid cancer, promoter hypermethylation of DAPK was demonstrated in $34 \%$ of PTC and was associated with tumor multifocality (51\%) [45].

3.7. Retinoic Acid Receptor- $\beta 2$ (RAR $\beta 2$ ). RAR $\beta 2$ plays a central role in the regulation of epithelial cell growth and tumorigenesis. Effects of retinoids are mediated by nuclear receptors, RAR- $\alpha$, RAR- $\beta$, and RAR- $\gamma$, RXR- $\alpha$, RXR- $\beta$, and RXR- $\gamma$ which form RXR-RAR heterodimers, that bind to specific DNA sequences, called RAR elements. It is thought that decreased expression of RARs may lead to resistance to retinoid effects [53]. Hypermethylation of RAR $\beta 2$ was demonstrated in $22 \%$ of papillary thyroid cancer and was not associated with any aggressive clinicopathologic features [45].

\section{Conclusion}

With improved diagnostic techniques, endocrine tumors are identified much more frequently than in the past. For instance, benign parathyroid tumors occur in as many as $2.3 \%$ of postmenopausal women [54], and primary hyperaldosteronism may be the cause of hypertension in as many as $4.8 \%$ of all patients with elevated blood pressure [55]. The molecular genetics of rare inherited endocrine tumor susceptibility syndromes, such as multiple endocrine neoplasia (MEN) type 1 and 2, familial pheochromocytoma syndromes, Carney complex, and Beckwith-Wiedemann syndrome have all contributed to our understanding of endocrine tumor development [56, 57]. Among different tumor types, there exist common pathways that lead to tumorigenesis, such as inactivation of the MEN1 tumor suppressor and activation of the RET proto-oncogene. As in other cancers, it is believed that the vast majority of genetic changes are somatic, that is, tumor-specific mutations acquired during tumor progression [58].

Epigenetic mechanisms, especially aberrant DNA methylation, very likely play an important role in papillary thyroid tumorigenesis. Genome-wide DNA methylation studies in 
PTC provide a powerful tool to identify disease-causing genes. Additionally, unbiased, systematic analyses of tumor methylomes are likely to identify signaling pathways of importance in cancer development, in general. Analyzing epigenetic alterations in papillary thyroid cancer would help to characterize pathogenesis and may play a critical role in tumor classification and diagnosis. It has recently been shown that there are differences in global methylation profiles between prognostic subsets of chronic lymphocytic leukemia (CLL) [59]. The specific silencing of unmethylated tumor suppressor genes was seen in the unmutated IGHV subgroup of CLL, implying a critical role for epigenetic changes during leukemogenesis. Interestingly, patients with immunoglobulin heavy-chain variable gene (IGHV) unmutated CLL have worse prognoses compared to CLL patients with mutated IGHV genes [59].

These studies may also pave the way for the application of epigenetic therapeutics, by targeted reversal of gene silencing. Azanucleoside drugs are demethylating agents that are currently approved for treatment of myelodyspastic syndrome $[20,56]$. These function as DNA methyltransferase enzymes that require incorporation into DNA to be effective and affect the differentiated state. Other nucleoside DNA methylation inhibitors include 5-fluoro-2'-deoxycytidine and zebulamine [60] which are in development. Histone deacetylases [61, 62] and histone methyltransferases are another reasonable option for therapeutics. The histone deacetylase SAHA is currently approved by the FDA for treatment of Tcell lymphoma [63].

Just as multiple epigenetic events may act in concert to affect a single-cell pathway, it is most likely that epigenetic therapy will involve using multiple drugs that individually affect epigenetic silencing but have synergistic effects. Proposed strategies for the FDA approved epigenetic drugs are as single therapies or in combination as primary or secondary treatment after neoadjuvant chemotherapy. Lack of specificity may not pose a problem, since DNMT inhibitors act only on dividing cells. The drugs preferentially activate genes that have become abnormally silenced in cancer [64]. Moreover, the chromatin structure associated with a pathologically silenced gene may be more susceptible to reactivation than the highly compacted state induced by physiological silencing [20].

\section{References}

[1] A. Jemal, R. Siegel, J. Xu, and E. Ward, "Cancer statistics," Cancer Journal for Clinicians, vol. 60, pp. 277-300, 2010.

[2] L. Davies and H. G. Welch, "Increasing incidence of thyroid cancer in the United States, 1973-2002," Journal of the American Medical Association, vol. 295, no. 18, pp. 2164-2167, 2006.

[3] M. L. Urken, "Prognosis and management of invasive welldifferentiated thyroid cancer," Otolaryngologic Clinics of North America, vol. 43, no. 2, pp. 301-328, 2010.

[4] M. Xing, "BRAF mutation in thyroid cancer," EndocrineRelated Cancer, vol. 12, pp. 245-262, 2005.

[5] Y. E. Nikiforov, "RET/PTC rearrangement in thyroid tumors," Endocrine Pathology, vol. 13, pp. 3-16, 2002.
[6] R. Ciampi and Y. E. Nikiforov, "RET/PTC rearrangements and BRAF mutations in thyroid tumorigenesis," Endocrinology, vol. 148, pp. 936-941, 2007.

[7] M. Xing, "Genetic alterations in the phosphatidylinositol-3 kinase/Akt pathway in thyroid cancer," Thyroid, vol. 20, pp. 697-706, 2010.

[8] H. Davies, G. N. Ranzani, P. Bianchi et al., "Mutations of the BRAF gene in human cancer," Nature, vol. 417, pp. 949-954, 2002.

[9] X. Xu, R. M. Quiros, P. Gattuso, K. B. Ain, and R. A. Prinz, "High prevalence of BRAF gene mutation in papillary thyroid carcinomas and thyroid tumor cell lines," Cancer Research, vol. 19, pp. 4561-4567, 2003.

[10] E. T. Kimura, E. Rosenbaum, K. J. Rhoden et al., "High prevalence of BRAF mutations in thyroid cancer: genetic evidence for constitutive activation of the RET/PTC-RASBRAF signaling pathway in papillary thyroid carcinoma," Cancer Research, vol. 63, pp. 1454-1457, 2003.

[11] M. N. Nikiforova, E. T. Kimura, M. Gandhi et al., "BRAF mutations in thyroid tumors are restricted to papillary carcinomas and anaplastic or poorly differentiated carcinomas arising from papillary carcinomas," Journal of Clinical Endocrinology and Metabolism, vol. 88, no. 11, pp. 5399-5404, 2003.

[12] V. Vasko, S. Hu, G. Wu et al., "High prevalence and possible de novo formation of BRAF mutation in metastasized papillary thyroid cancer in lymph nodes," Journal of Clinical Endocrinology and Metabolism, vol. 90, no. 9, pp. 5265-5269, 2005.

[13] M. Xing, W. H. Westra, R. P. Tufano et al., "BRAF mutation predicts a poorer clinical prognosis for papillary thyroid cancer," Journal of Clinical Endocrinology and Metabolism, vol. 90, no. 12, pp. 6373-6379, 2005.

[14] M. Rivera, W. H. Westra, R. P. Tufano et al., "Molecular, morphologic, and outcome analysis of thyroid carcinomas according to degree of extrathyroid extension," Thyroid, vol. 20, pp. 1085-1093, 2010.

[15] T. J. Musholt, W. H. Westra, R. P. Tufano et al., "Detection of papillary thyroid carcinoma by analysis of BRAF and RET/PTC1 mutations in fine-needle aspiration biopsies of thyroid nodules," World Journal of Surgery, vol. 34, pp. 25952603, 2010.

[16] V. Vasko, M. Ferrand, J. Di Cristofaro, P. Carayon, J. F. Henry, and C. De Micco, "Specific pattern of RAS oncogene mutations in follicular thyroid tumors," Journal of Clinical Endocrinology and Metabolism, vol. 88, no. 6, pp. 2745-2752, 2003.

[17] T. J. Musholt, W. H. Westra, R. P. Tufano et al., "Impact of pathognomonic genetic alterations on the prognosis of papillary thyroid carcinoma. ESES vienna presentation," Langenbeck Archives of Surgery, vol. 395, pp. 877-883, 2010.

[18] E. Ishida, M. Nakamura, K. Shimada et al., "DNA hypermethylation status of multiple genes in papillary thyroid carcinomas," Pathobiology, vol. 74, no. 6, pp. 344-352, 2007.

[19] H. Zuo, M. Gandhi, M. M. Edreira et al., "Downregulation of Rap1GAP through epigenetic silencing and loss of heterozygosity promotes invasion and progression of thyroid tumors," Cancer Research, vol. 70, no. 4, pp. 1389-1397, 2010.

[20] P. A. Jones and S. B. Baylin, "The Epigenomics of Cancer," Cell, vol. 128, no. 4, pp. 683-692, 2007.

[21] M. Esteller, M. F. Fraga, M. Guo et al., "DNA methylation patterns in hereditary human cancers mimic sporadic tumorigenesis," Human Molecular Genetics, vol. 10, no. 26, pp. 30013007, 2001. 
[22] A. P. Feinberg, R. Ohlsson, and S. Henikoff, "The epigenetic progenitor origin of human cancer," Nature Reviews Genetics, vol. 7, no. 1, pp. 21-33, 2006.

[23] S. B. Baylin and J. E. Ohm, "Epigenetic gene silencing in cancer-a mechanism for early oncogenic pathway addiction?” Nature Reviews Cancer, vol. 6, no. 2, pp. 107-116, 2006.

[24] A. Eden, F. Gaudet, A. Waghmare, and R. Jaenisch, "Chromosomal instability and tumors promoted by DNA hypomethylation," Science, vol. 300, no. 5618, p. 455, 2003.

[25] J. G. Herman and S. B. Baylin, "Gene silencing in cancer in association with promoter hypermethylation," New England Journal of Medicine, vol. 349, no. 21, pp. 2042-2054, 2003.

[26] C. A. Iacobuzio-Donahue, "Epigenetic changes in cancer," Annual Review of Pathology, vol. 4, pp. 229-249, 2009.

[27] M. Esteller, A. Sparks, M. Toyota et al., "Analysis of adenomatous polyposis coli promoter hypermethylation in human cancer," Cancer Research, vol. 60, no. 16, pp. 4366-4371, 2000.

[28] S. A. Foster, D. J. Wong, M. T. Barrett, and D. A. Galloway, "Inactivation of p16 in human mammary epithelial cells by CpG island methylation," Molecular and Cellular Biology, vol. 18, pp. 1793-1801, 1998.

[29] T. Kiyono, S. A. Foster, J. I. Koop, J. K. McDougall, D. A. Galloway, and A. J. Klingelhutz, "Both Rb/p16INK4a inactivation and telomerase activity are required to immortalize human epithelial cells," Nature, vol. 396, no. 6706, pp. 84-88, 1998.

[30] P. A. Reynolds, M. Sigaroudinia, G. Zardo et al., "Tumor suppressor p16 regulates polycomb-mediated DNA hypermethylation in human mammary epithelial cells," Journal of Biological Chemistry, vol. 281, no. 34, pp. 24790-24802, 2006.

[31] Y. Akiyama, N. Watkins, H. Suzuki et al., "GATA-4 and GATA-5 transcription factor genes and potential downstream antitumor target genes are epigenetically silenced in colorectal and gastric cancer," Molecular and Cellular Biology, vol. 23, no. 23, pp. 8429-8439, 2003.

[32] X. Nan, H. H. Ng, C. A. Johnson et al., "Transcriptional repression by the methyl-CpG-binding protein MeCP2 involves a histone deacetylase complex," Nature, vol. 393, no. 6683, pp. 386-389, 1998.

[33] P. L. Jones, G. J. C. Veenstra, P. A. Wade et al., "Methylated DNA and MeCP2 recruit histone deacetylase to repress transcription," Nature Genetics, vol. 19, no. 2, pp. 187-191, 1998.

[34] A. P. Feinberg and B. Tycko, "The history of cancer epigenetics," Nature Reviews Cancer, vol. 4, no. 2, pp. 143-153, 2004.

[35] M. O. Hoque, E. Rosenbaum, W. H. Westra et al., "Quantitative assessment of promoter methylation profiles in thyroid neoplasms," Journal of Clinical Endocrinology and Metabolism, vol. 90, no. 7, pp. 4011-4018, 2005.

[36] M. Xing, H. Usadel, Y. Cohen et al., "Methylation of the thyroid-stimulating hormone receptor gene in epithelial thyroid tumors: a marker of malignancy and a cause of gene silencing," Cancer Research, vol. 63, no. 9, pp. 2316-2321, 2003.

[37] J. A. Smith, C. Y. Fan, C. Zou, D. Bodenner, and M. S. Kokoska, "Methylation status of genes in papillary thyroid carcinoma," Archives of Otolaryngology, vol. 133, no. 10, pp. 1006-1011, 2007.

[38] G. M. Venkataraman, M. Yatin, R. Marcinek, and K. B. Ain, "Restoration of iodide uptake in dedifferentiated thyroid carcinoma: relationship to human $\mathrm{Na}+/ \mathrm{I}$ symporter gene methylation status," Journal of Clinical Endocrinology and Metabolism, vol. 84, no. 7, pp. 2449-2457, 1999.
[39] J. R. Graff, V. E. Greenberg, J. G. Herman et al., "Distinct patterns of E-cadherin CpG island methylation in papillary, follicular, hurthle's cell, and poorly differentiated human thyroid carcinoma," Cancer Research, vol. 58, no. 10, pp. 20632066, 1998.

[40] S. Banin, L. Moyal, S. Y. Shieh et al., "Enhanced phosphorylation of p53 by ATM in response to DNA damage," Science, vol. 281, no. 5383, pp. 1674-1677, 1998.

[41] C. E. Canman, D. S. Lim, K. A. Cimprich et al., "Activation of the ATM kinase by ionizing radiation and phosphorylation of p53," Science, vol. 281, no. 5383, pp. 1677-1679, 1998.

[42] D. Cortez, Y. Wang, J. Qin, and S. J. Elledge, "Requirement of ATM-dependent phosphorylation of Brcal in the DNA damage response to double-strand breaks," Science, vol. 286, no. 5442, pp. 1162-1166, 1999.

[43] L. Lacroix, T. Pourcher, C. Magnon et al., "Expression of the apical iodide transporter in human thyroid tissues: a comparison study with other iodide transporters," Journal of Clinical Endocrinology and Metabolism, vol. 89, no. 3, pp. 1423-1428, 2004.

[44] V. Porra, C. Ferraro-Peyret, C. Durand et al., "Silencing of the tumor suppressor gene SLC5A8 is associated with BRAF mutations in classical papillary thyroid carcinomas," Journal of Clinical Endocrinology and Metabolism, vol. 90, no. 5, pp. 3028-3035, 2005.

[45] S. Hu, D. Liu, R. P. Tufano et al., "Association of aberrant methylation of tumor suppressor genes with tumor aggressiveness and BRAF mutation in papillary thyroid cancer," International Journal of Cancer, vol. 119, no. 10, pp. 23222329, 2006.

[46] J. H. Qi, Q. Ebrahem, N. Moore et al., "A novel function for tissue inhibitor of metalloproteinases-3 (TIMP3): inhibition of angiogenesis by blockage of VEGF binding to VEGF receptor-2," Nature Medicine, vol. 9, no. 4, pp. 407-415, 2003.

[47] S. J. Darnton, L. J. Hardie, R. S. Muc, C. P. Wild, and A. G. Casson, "Tissue inhibitor of metalloproteinase-3 (TIMP3) gene is methylated in the development of esophageal adenocarcinoma: loss of expression correlates with poor prognosis," International Journal of Cancer, vol. 115, no. 3, pp. 351-358, 2005.

[48] H. Feng, A. N. Y. Cheung, W. C. Xue et al., "Down-regulation and promoter methylation of tissue inhibitor of metalloproteinase 3 in choriocarcinoma," Gynecologic Oncology, vol. 94, no. 2, pp. 375-382, 2004.

[49] P. A. Van der Velden, W. Zuidervaart, M. H. M. H. Hurks et al., "Expression profiling reveals that methylation of TIMP3 is involved in uveal melanoma development," International Journal of Cancer, vol. 106, no. 4, pp. 472-479, 2003.

[50] R. Schneider-Stock, A. Roessner, and O. Ullrich, "DAPkinase-protector or enemy in apoptotic cell death," International Journal of Biochemistry and Cell Biology, vol. 37, no. 9, pp. 1763-1767, 2005.

[51] A. Satoh, M. Toyota, F. Itoh et al., "DNA methylation and histone deacetylation associated with silencing DAP kinase gene expression in colorectal and gastric cancers," British Journal of Cancer, vol. 86, no. 11, pp. 1817-1823, 2002.

[52] S. V. Harden, M. Toyota, F. Itoh et al., "Gene promoter hypermethylation in tumors and lymph nodes of stage I lung cancer patients," Clinical Cancer Research, vol. 9, pp. 13701375, 2003.

[53] E. M. Youssef, D. Lotan, J. P. Issa et al., "Hypermethylation of the retinoic acid receptor- $\beta$ gene in head and neck carcinogenesis," Clinical Cancer Research, vol. 10, no. 5, pp. 1733-1742, 2004. 
[54] E. Lundgren, J. Rastad, E. Thurfjell, G. Akerstrom, and S. Ljunghall, "Population-based screening for primary hyperparathyroidism with serum calcium and parathyroid hormone values in menopausal women," Surgery, vol. 121, no. 3, pp. 287-294, 1997.

[55] G. P. Rossi, G. Bernini, C. Caliumi et al., "A prospective study of the prevalence of primary aldosteronism in 1,125 hypertensive patients," Journal of the American College of Cardiology, vol. 48, no. 11, pp. 2293-2300, 2006.

[56] T. Carling, "Molecular pathology of parathyroid tumors," Trends in Endocrinology and Metabolism, vol. 12, no. 2, pp. 5358, 2001.

[57] T. Carling, "Multiple endocrine neoplasia syndrome: genetic basis for clinical management," Current Opinion in Oncology, vol. 17, no. 1, pp. 7-12, 2005.

[58] M. R. Stratton, P. J. Campbell, and P. A. Futreal, "The cancer genome," Nature, vol. 458, no. 7239, pp. 719-724, 2009.

[59] M. Kanduri, N. Cahill, H. Göransson et al., "Differential genome-wide array-based methylation profiles in prognostic subsets of chronic lymphocytic leukemia," Blood, vol. 115, no. 2, pp. 296-305, 2010.

[60] J. C. Cheng, C. B. Yoo, D. J. Weisenberger et al., "Preferential response of cancer cells to zebularine," Cancer Cell, vol. 6, no. 2, pp. 151-158, 2004.

[61] P. Marks, R. A. Rifkind, V. M. Richon, R. Breslow, T. Miller, and W. K. Kelly, "Histone deacetylases and cancer: causes and therapies," Nature Reviews Cancer, vol. 1, no. 3, pp. 194-202, 2001.

[62] S. Minucci and P. G. Pelicci, "Histone deacetylase inhibitors and the promise of epigenetic (and more) treatments for cancer," Nature Reviews Cancer, vol. 6, no. 1, pp. 38-51, 2006.

[63] J. E. Bolden, M. J. Peart, and R. W. Johnstone, "Anticancer activities of histone deacetylase inhibitors," Nature Reviews Drug Discovery, vol. 5, no. 9, pp. 769-784, 2006.

[64] G. Liang, M. L. Gonzalgo, C. Salem, and P. A. Jones, "Identification of DNA methylation differences during tumorigenesis by methylation-sensitive arbitrarily primed polymerase chain reaction," Methods, vol. 27, no. 2, pp. 150-155, 2002. 


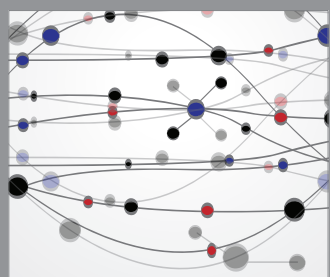

The Scientific World Journal
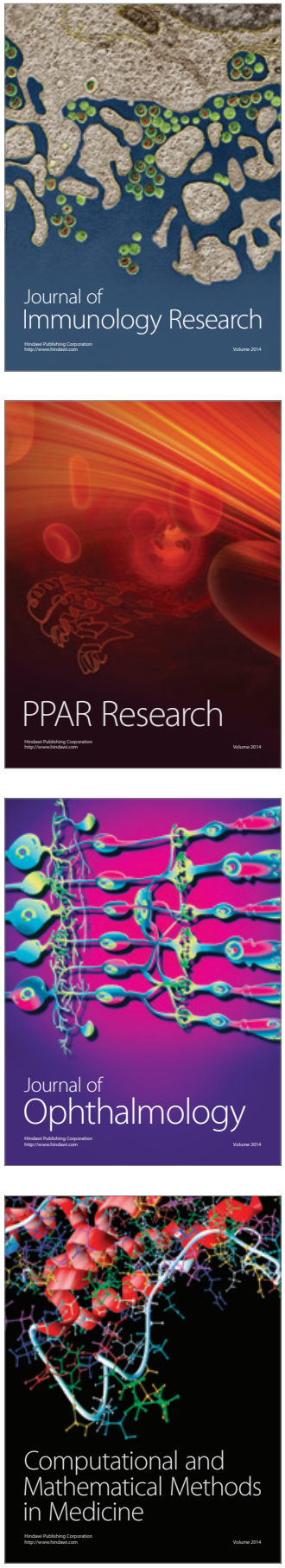

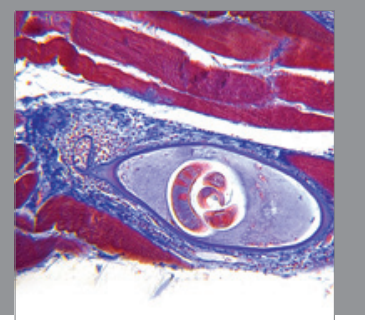

Gastroenterology

Research and Practice
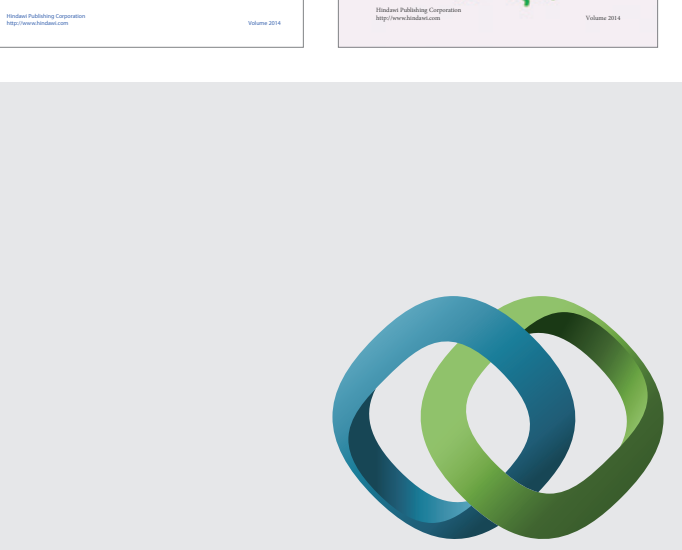

\section{Hindawi}

Submit your manuscripts at

http://www.hindawi.com
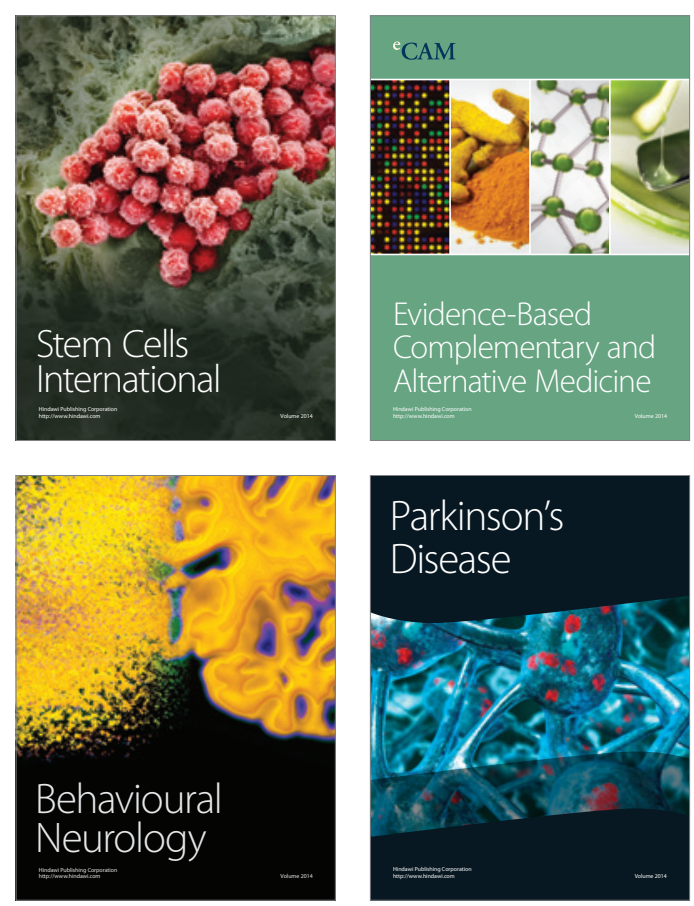

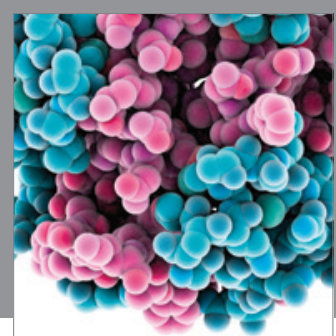

Journal of
Diabetes Research

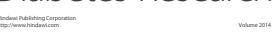

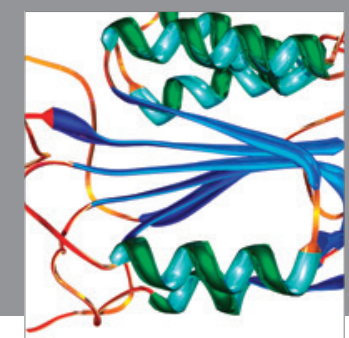

Disease Markers
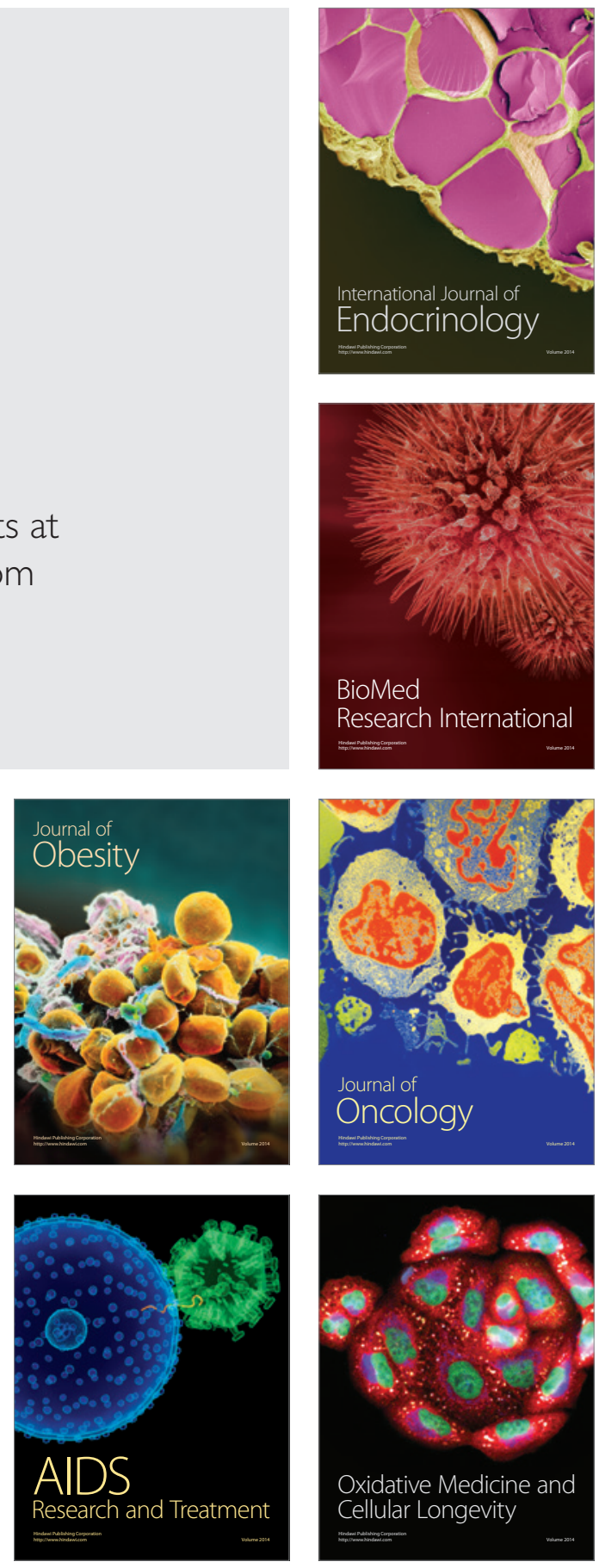